\section{Controversies in Surgery}

Editors: M. Schein, L. Wise

Berlin, Springer, 2001

395 p., 43 fig., 3 tab., EUR 99,95; US\$ 95,00

ISBN 3-540-41103-8

Das Buch ist der vierte Band einer Serie, die 1994 von den Autoren gestartet wurde. Das Prinzip der Wissensvermittlung mit Hilfe der Darstellung von Kontroversen bei unterschiedlichen Themen aus der Viszeral- und Gefäßchirurgie wird konsequent beibehalten. Dabei werden kontroverse Themenbereiche in den einzelnen Kapiteln stichpunktartig aufgelistet, um von den jeweiligen Autoren, den eingeladenen Kommentatoren und abschließend von den Herausgebern selbst dargestellt und gewertet zu werden. Die Praxisnähe dieses Buches zeigt sich z.B. im Kapitel über akute Cholecystitis. Folgende Kontroversen werden aufgezählt: intraoperative Cholangiographie, operative Verfahrensweise und Zeitpunkt der Operation, akute Cholecystitis und Schwangerschaft, akute Cholecystitis und Diabetes mellitus und akute Cholecystitis bei Hochrisikopatienten. Auf diese Weise werden verschiedene gutartige und bösartige Erkrankungen, aber auch Themen wie akute Appendizitis, mesenteriale Durchblutungsstörungen, Gefäßverletzungen und Adipositas permagna abgehandelt. Ein ausführliches Kapitel ist der laparoskopischen Chirurgie gewidmet. Neben diesen gängigen Themen finden sich aber auch Beiträge, die in klinisch betonten Chirurgiebüchern seltener sind. So beginnt das Buch mit einem Kapitel über das Publizieren auf dem Gebiet der Chirurgie im 21. Jahrhundert. Hier werden die modernen Medien gegeneinander abgewogen, wobei trotz der «unbegrenzten» Möglichkeiten des Internets das klassische Buch nach Ansicht mehrerer Autoren seinen Stellenwert behalten wird. Das Buch schließt mit einem Kapitel über chirurgische Fehler ab, wobei die Befindlichkeiten aus Sicht von Chirurgen, Patienten und Juristen sehr einfühlsam dargestellt werden. Die Autoren dieses Buches kommen überwiegend aus den USA, England und anderen europäischen Staaten. Die unterschiedlichen Mentalitäten finden sich auch in fachlichen Darstellungen wieder. Zur richtigen Einschätzung der unterschiedlichen Meinungen ist es von Nutzen, wenn man einige Erfahrungen auf internationalen Kongressen gemacht hat. Meines Erachtens ist das Buch deshalb auch eher für den erfahrenen Kliniker geeignet, der mit einem gewissen Abstand an die Themen herangeht und nicht mehr oder weniger unkritisch Wissen in sich aufnehmen will oder muss. Es entspricht den Anforderungen einer Evidence- basierten Medizin, wobei einige Kapitel mit 70 bis über 1000 Literaturstellen etwas überfrachtet erscheinen. Sein Preis ist angemessen.

R. Kirchner, Hannover

\section{Praxis der enteralen Ernährung. Indikation - Technik - Nachsorge}

Herausgeber: C. Löser, M. Keymling

Stuttgart, Thieme, 2001

296 S., 88 Tab., 197 Abb., EUR 79,95; SFr 131,00

ISBN 3-13-129261-X

Der signifikante Einfluss einer frühzeitigen Diagnostik und Therapie der Mangelernährung auf die individuelle Komplikationsrate, Therapietoleranz, Morbidität, Mortalität und damit auf die generellen Prognose der Patienten wird auch heute nicht selten unterschätzt. Dabei wurden die Erfolge der nutritiven Therapie in der Onkologie sowie bei neurologischen und geriatrischen Patienten nach Einführung der perkutanen endoskopischen Gastrostomie (PEG) in Deutschland offensichtlich. Das Buch wendet sich fachübergreifend an alle in Klinik und Praxis tätigen Ärzte, Schwestern und Pfleger, die mit der künstlichen Ernährung befasst sind. Es stellt eine aktuelle Zwischenbilanz unter Berücksichtigung aktueller Studienergebnisse dar.

Der Inhalt dieses Buches ist übersichtlich gegliedert. Am Beginn stehen physiologische Grundlagen der Ernährung sowie die Pathophysiologie der Malnutrition. Es folgt eine kritische Darstellung der Vor- und Nachteile enteraler und parenteraler Ernährungsstrategien bei kritisch Kranken, wobei besonders auch immunologische Aspekte berücksichtigt werden. Wertvolle Hinweise für die praktische Durchführung der enteralen Ernährung finden sich in den Kapiteln über Techniken der Sondeneinlage, die das gesamte Spektrum von der einfachen nasogastralen oder nasojejunalen Sonde über perkutan endoskopisch, laparoskopisch oder radiologisch platzierte Sonden hin zur operativen Anlage von Ernährungsfisteln einbeziehen. Dabei werden neben Indikation, Kontraindikation und Komplikationen auch ethische und juristische Aspekte dargestellt. Danach wird die enterale Ernährung, als Teil multimodaler Therapiekonzepte perioperativ, bei Tumorerkrankungen, in der Geriatrie und Pädiatrie sowie auf Intensivstation abgehandelt. Ausreichend Raum wird auch der Pflege und Langzeitbetreuung von Patienten mit enteralen Sondensystemen 
gewidmet. Kasuistiken und ein tabellarischer Anhang über Produkte und Hersteller runden das Ganze ab.

Insgesamt spricht das Buch eine für manchen Kliniker nicht vertraute Materie an und stellt sie anschaulich mit vielen, zum großen Teil farbigen Abbildungen und didaktisch gelungenen Tabellen auf dem neuesten wissenschaftlichen Stand dar. Es ist von Autoren verschiedener Fachrichtungen und unterschiedlicher Institutionen verfasst und für alle, die mit der enteralen Ernährung konfrontiert sind, eine sowohl theoretische als auch sehr praktische Hilfe.

R. Kirchner, Hannover

\section{Klinikleitfaden Chirurgie}

3. Aufl.

Herausgeber: F.-M. Hasse, H. Nürnberger

München, Urban \& Fischer, 2002

1072 S., 250 Abb., 195 Tab., EUR 39,95, SFr 69,20

ISBN 3-437-22450-6

Die Herausgeber haben mit weiteren 33 Autoren aus ganz Deutschland die 3. Auflage des bewährten Klinikleitfadens Chirurgie vorgelegt, der in der Kitteltasche getragen werden kann und sich hervorragend für die Arbeit in der chirurgischen Klinik eignet. Das Buch ist kurz, präzise und praxisnah aufgebaut. Im Zentrum des komprimiert dargestellten medizinischen Wissens stehen die Probleme des klinischen Alltags. Deswegen wird auf die Darstellung theoretischer Grundlagen wie Pathophysiologie und allgemeine Pharmakologie weitgehend verzichtet. Um Wiederholungen zu vermeiden, sind viele Querverweise eingefügt, die mit einer weisenden Hand hervorgehoben sind. Als weitere bildliche Hinweise finden sich Ausrufungszeichen für wichtige Zusatzinformationen, Blitzzeichen für Notfälle und -maßnahmen und das Bild einer Bombe für Warnhinweise. Das Buch ist in Tipps für die Stationsarbeit, Hinweise auf chirurgische Arbeitstechniken, den chirurgischen Eingriff, häufige internistische und neurologische Probleme, Begutachtung und apparative Diagnostik, vor allem Röntgen, Sonographie und Verfahren der Endoskopie, Darstellung der Grundlagen der chirurgischen Notfall- und Intensivtherapie, Transplantationschirurgie, praktische Onkologie und Infektionen nach Organsystemen gegliedert. Die Kapitel 30-33 behandeln Probleme der Arzneitherapie, Referenzbereiche und Differenzialdiagnose pathologischer Laborparameter, wichtige Adressen mit Internetangabe (Redaktionsschluss November 2001) und die AO-Klassifikation langer Röhrenknochen. Zum Schluss folgt ein ausführliches Sachverzeichnis. Der Klinikleitfaden eignet sich hervorragend für den chirurgischen Assistenten. Er ist didaktisch sehr gut aufgebaut, zweifarbig, trotz der kleinen Schrift gut lesbar und verständlich. Dazu tragen hervorragend reproduzierte Abbildungen, teilweise als Schemazeichnungen, bei. Inhaltlich sind vereinzelt unbedeutende Mängel aufgefallen, die jedoch bei einem so umfangreichen Werk vernachlässigt werden können. Der Klinikleitfaden ist daher ohne Einschränkung zu empfehlen. Sein Preis ist sehr günstig.

K.-J. Paquet, Hannover

\section{Funktionsdiagnostik in der Gastroenterologie. Medizinische Standards}

Herausgeber: J. Stein, T. Wehrmann

Berlin, Springer, 2002

358 S., 87 Abb., 81 Tab., EUR 34,95

ISBN 3-540-67391-1

Die Herausgeber sind ausgewiesene Endoskopiker und Gastroenterologen, die für ihr Engagement für die Funktionsdiagnostik in der Gastroenterologie bekannt sind. Mit wenigen Mitarbeitern haben sie dieses umfangreiche Gebiet in fünf Kapiteln gründlich abgehandelt. Sie beginnen mit dem oberen Gastrointestinaltrakt, woran sich der untere Gastrointestinaltrakt, Funktionstests für Pankreas, Leber und Galle und solche für Leitsymptome, wie z.B. Obstipation, anschließen. Das umfangreiche Taschenbuch enthält alle wesentlichen Aspekte der Thematik und ist durch Zweifarbigkeit bei Merksätzen, guten Schwarzweiß- bzw. farbigen Abbildungen und unterschiedliche Schriftbilder illustriert. Jedes Kapitel enthält ein kurzes Literaturverzeichnis. Das Sachverzeichnis ist für das umfangreiche Buch etwas kurz geraten. Trotz dieses kleinen Mangels kann dieses hervorragende Taschenbuch als Standardwerk für die Thematik insbesondere den Gastroenterologen, jedoch auch Viszeralchirurgen, zur bereichernden Lektüre empfohlen werden. Sein Preis ist angemessen.

K.-J. Paquet, Hannover

\section{Das hepatozelluläre Karzinom}

Herausgeber: H.-P. Allgaier

Berlin, UNI-MED, 2002

126 S., 79 z.T. farbige Abb., 26 Tab., EUR 44,80

ISBN 3-89599-607-6

Die Science-Reihe des Verlags UNI-MED stellt aktuelle Forschungsergebnisse zur Diagnostik und Therapie wichtiger Erkrankungen dar, die sich durch höchste wissenschaftliche Kompetenz und anspruchsvolle Präsentation auszeichnen sollen. Die Autoren werden als Meinungsbilder auf ihren Fachgebieten bezeichnet. Das ist ein sehr hoher Anspruch an das Buch und seinen Herausgeber. Als Mitautoren hat er zehn seiner Kolleginnen und Kollegen der Medizinischen Universitätsklinik II und des Pathologischen Instituts der Universität Freiburg - einschließlich seines Lehrers und Chefs, Hubert E. Blum - und zwei Chirurgen des Universitätsklinikums Charitè Berlin, Virchow-Klinikum, gewinnen können. Das Buch hat 
folgende Kapitel: «Epidemiologie und Klinik», «Pathogenese», «Diagnostik», «HCC-Stadien» und «Prognose», «Therapie», «Gen-Therapie des HCC», «Zusammenfassung», «Perspektiven» sowie einen Sachindex. Es ist didaktisch hervorragend gestaltet, indem wichtige Aussagen farblich unterlegt und die Ausführungen durch sehr gute Farbabbildungen und Tabellen illustriert sind. Die Literaturverzeichnisse am Ende der Kapitel sind etwas spärlich ausgefallen. Gleiches gilt für den Index. Hinter beidem vermutet der Rezensent Zeitdruck. Aus diesen und auch anderen inhaltlichen Gründen wird dieses ansonsten hervorragende Buch dem genannten Anspruch, dass es höchste wissenschaftliche Kompetenz aufweist, nicht vollständig gerecht. Seine Lektüre wird jedoch jeden an der Thematik Interessierten, das sind Gastroenterologen, Hepatologen, Viszeralchirurgen, Pathologen und Radiolgen, bereichern. Sein Preis ist angemessen.

K.-J. Paquet, Hannover

\section{Sonographie für den Chirurgen}

Herausgeber: R. Mantke, U. Peitz

Stuttgart, Thieme, 2001

410 S., 914 Abb., 75 Tab., EUR 149,00, SFr 257,00

ISDN 3-131-24591-3

Der Chirurg R. Mantke und der Gastroenterologe und Hepatologe U. Peitz der Otto-von-Uericke-Universität Magdeburg haben sich zusammengefunden, um einen umfassenden Überblick über die Sonographie für den Chirurgen zu verfassen. Dieses Vorhaben ist ein Novum, denn es gibt auf dem Büchermarkt noch kein aktuelles Lehrbuch für die gesamte Sonographie, welches in umfassender und kompakter Weise alle Schwerpunkte der Chirurgie und den Wert der Sonographie für dieses Fachgebiet analysiert und darstellt. Von großer Bedeutung ist dabei, dass der Stellenwert der Sonographie ständig wächst, da sie eine hohe diagnostische Aussagekraft besitzt, überall, jederzeit, z.B. auch auf der Intensivstation, verfügbar ist, keine wesentlichen Nebenwirkungen aufweist und darüber hinaus sehr preisgünstig ist. An dem Lehrbuch sind außer den Herausgebern 31 Autoren, vorwiegend aus der Universitätsklinik Magdeburg, den Universitätskliniken
Essen, Marburg, Graz, Greifswald und Jena, sowie Kollegen, die in selbstständigen Positionen an Schwerpunktkrankenhäusern oder auch in einer orthopädischen Praxis arbeiten, beteiligt. Da die Sonographie eine interdisziplinäre Wissenschaft darstellt, sind außer Chirurgen und Internisten Orthopäden, Pädiater, Urologen, Gynäkologen und Radiologen um diese Publikation bemüht. Das Buch ist in die Kapitel «Akutes Abdomen», «Thorax- und Abdominaltrauma», «Intraoperative Sonographie in der Chirurgie von Leber, Pankreas und im Rahmen einer Staging-Operation», «Sonographie auf der Intensivstation», «Interventionelle Sonographie», «Technische Grundlagen», «Abdominalsonographie», «Sonographie von Pleura und Thorax, Schilddrüse und des endokrinen Systems», „Endosonographie», «Sonographie von Niere, harnableitendem System und Scrotum, der weiblichen Brustdrüse, der Gelenke, Knochen und angrenzenden Weichteile, der Bauchdecke, der Arterien und Venen», «Sonographie nach Leber-, Nieren- oder Pankreastransplantation, der intra-abdominellen Farb-Duplex-Sonographie der Arterien und der Kinderchirurgischen Sonographie» aufgeteilt. Es beginnt mit einem Abkürzungs- und endet mit einem Sachverzeichnis. Die Kapitel enthalten hervorragende, teilweise farbige sonographische Abbildungen, denen ein Schema und wo die Autoren es für nötig halten farbige Operationsbilder, farbige Schemen der Anatomie und zweifarbige Tabellen zur Seite gestellt sind. Insgesamt ist ein in jeder Hinsicht hervorragendes, lesenswertes und didaktisch optimal gelungenes Lehrbuch über die Sonographie für den Chirurgen entstanden, das jedem jungen Chirurgen, aber auch den interdisziplinär mit der Chirurgie kooperierenden anderen Fachdisziplinen zum Studium empfohlen werden kann. Von seiner Lektüre werden auch erfahrene Fachärzte der Chirurgie und insbesondere solche in selbstständigen Positionen profitieren, die auf diesem Gebiet ein Bedürfnis, Informationen nachzuholen, besitzen dürften. Es ist ihm daher eine weite Verbreitung in diesem Personenkreis zu wünschen. $\mathrm{Zu}$ Recht fordern die Deutsche Gesellschaft für Chirurgie und auch die Ärztekammern im Rahmen der Facharztweiterbildung, dass die Sonographie heute von jedem Chirurgen beherrscht werden muss. Dazu kann ihm nur ein sorgfältiges und intensives Studium z.B. eines solchen Standardwerks verhelfen. Sein Preis ist in Anbetracht der hervorragenden Ausstattung günstig. 